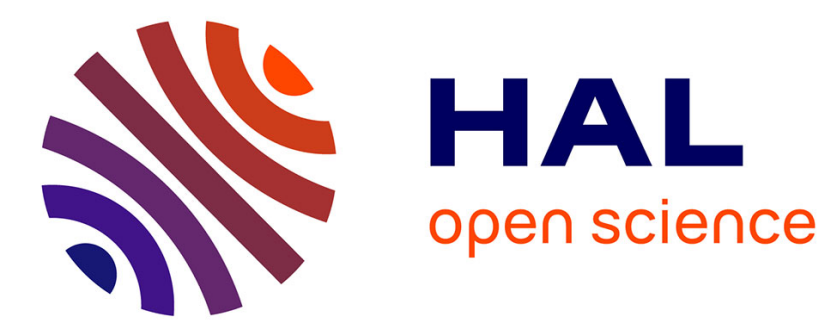

\title{
On a homogenization technique for singular integrals
}

\author{
Omar Anza Hafsa, Mohammed Lamine Leghmizi, Jean-Philippe Mandallena
}

\section{To cite this version:}

Omar Anza Hafsa, Mohammed Lamine Leghmizi, Jean-Philippe Mandallena. On a homogenization technique for singular integrals. Asymptotic Analysis, 2011, 74 (3-4), pp.123-134. 10.3233/ASY2011-1042 . hal-00797473

\section{HAL Id: hal-00797473 \\ https://hal.science/hal-00797473}

Submitted on 6 Mar 2013

HAL is a multi-disciplinary open access archive for the deposit and dissemination of scientific research documents, whether they are published or not. The documents may come from teaching and research institutions in France or abroad, or from public or private research centers.
L'archive ouverte pluridisciplinaire HAL, est destinée au dépôt et à la diffusion de documents scientifiques de niveau recherche, publiés ou non, émanant des établissements d'enseignement et de recherche français ou étrangers, des laboratoires publics ou privés. 


\title{
ON A HOMOGENIZATION TECHNIQUE FOR SINGULAR INTEGRALS
}

\author{
OMAR ANZA HAFSA, MOHAMED LAMINE LEGHMIZI, \\ AND JEAN-PHILIPPE MANDALLENA
}

AbStRaCt. We study homogenization by $\Gamma$-convergence of functionals of type

$$
\int_{\Omega} W\left(\frac{x}{\varepsilon}, \nabla \phi(x)\right) d x
$$

where $\Omega \subset \mathbb{R}^{N}$ is a bounded open set, $\phi \in W^{1, p}\left(\Omega ; \mathbb{R}^{m}\right)$ and $p>1$, when the 1-periodic integrand $W: \mathbb{R}^{N} \times \mathbb{M}^{m \times N} \rightarrow[0,+\infty]$ is not of $p$-polynomial growth. Our homogenization technique can be applied when $m=N$ and $W$ has a singular behavior of type $W(x, \xi) \rightarrow+\infty$ as $\operatorname{det} \xi \rightarrow 0$. However, our technique is not consistent with the constraint $W(x, \xi)=+\infty$ if and only if $\operatorname{det} \xi \leq 0$.

\section{INTRODUCTION}

Consider the family of integral functionals $\left\{I_{\varepsilon}\right\}_{\varepsilon>0}$ given by

$$
I_{\varepsilon}(\phi):=\int_{\Omega} W\left(\frac{x}{\varepsilon}, \nabla \phi(x)\right) d x,
$$

where $\varepsilon>0$ is a (small) parameter, $\Omega \subset \mathbb{R}^{N}$ a bounded open set with $|\partial \Omega|=0$ (where $|\cdot|$ denotes the Lebesgue measure in $\left.\mathbb{R}^{N}\right), \phi \in W^{1, p}\left(\Omega ; \mathbb{R}^{m}\right)$ with $p>1$ and $W: \mathbb{R}^{N} \times \mathbb{M}^{m \times N} \rightarrow[0,+\infty]$ assumed to be Borel measurable, where $\mathbb{M}^{m \times N}$ denotes the space of real $m \times N$ matrices, is $p$-coercive, i.e.,

$$
W(x, \xi) \geq C|\xi|^{p} \text { for all }(x, \xi) \in \mathbb{R}^{N} \times \mathbb{M}^{m \times N} \text { and some } C>0,
$$

1-periodic, i.e.,

$$
W\left(x+e_{i}, \xi\right)=W(x, \xi) \text { for all }(x, \xi) \in \mathbb{R}^{N} \times \mathbb{M}^{m \times N} \text { and } i=1, \cdots, N,
$$

where $\left(e_{1}, \cdots, e_{N}\right)$ is the standard basis of $\mathbb{R}^{N}$, and of $p$-polynomial growth, i.e.,

$$
W(x, \xi) \leq c\left(1+|\xi|^{p}\right) \text { for all }(x, \xi) \in \mathbb{R}^{N} \times \mathbb{M}^{m \times N} \text { and some } c>0 .
$$

In [Bra85] (see also [BD98, Theorem 14.5 p. 111]) Braides proved that $I_{\varepsilon} \Gamma$-converge with respect to the $L^{p}\left(\Omega ; \mathbb{R}^{m}\right)$-convergence as $\varepsilon \rightarrow 0$ (see Definition 3.1) to the functional $I_{\text {hom }}$ defined on $W^{1, p}\left(\Omega ; \mathbb{R}^{m}\right)$ by

$$
I_{\text {hom }}(\phi):=\int_{\Omega} W_{\text {hom }}(\nabla \phi(x)) d x
$$

with $W_{\text {hom }}: \mathbb{M}^{m \times N} \rightarrow[0,+\infty]$ given by

$$
W_{\text {hom }}(\xi):=\inf _{k \geq 1} \frac{1}{k^{N}} \inf \left\{\int_{k Y} W(x, \xi+\nabla \varphi(x)) d x: \varphi \in W_{0}^{1, p}\left(k Y ; \mathbb{R}^{m}\right)\right\}
$$

with $Y:=] 0,1{ }^{N}$ and $W_{0}^{1, p}\left(k Y ; \mathbb{R}^{m}\right):=\left\{\varphi \in W^{1, p}\left(k Y ; \mathbb{R}^{m}\right): \varphi=0\right.$ on $\left.\partial(k Y)\right\}$. This result established a suitable variational framework to deal with homogenization problems in the vectorial case: it is the point of departure of many works on the subject related to hyperelasticity (in the scalar case and for some problems

Key words and phrases. Homogenization, $\Gamma$-convergence, singular integrand, determinant constraints type, hyperelasticity. 
related to hyperelasticity, see [CCDAG02, CCDAG04]). However, because of the p-polynomial growth assumption (1.4), Braides's homogenization theorem is not compatible with the following two important physical properties: the noninterpenetration of the matter, i.e., $W(x, \xi)=+\infty$ if and only if $\operatorname{det} \xi \leq 0$, and the necessity of an infinite amount of energy to compress a finite volume into zero volume, i.e., $W(x, \xi) \rightarrow+\infty$ as $\operatorname{det} \xi \rightarrow 0$, where $\operatorname{det} \xi$ denotes the determinant of the $N \times N$ matrix $\xi$.

In this paper we show that by using Braides's homogenization theorem (see Theorem 3.2) and a slight generalization of a relaxation theorem (see Theorem 2.7), that we obtained in [AHM07, AHM08], it is possible to establish a homogenization theorem (see Theorem 3.4) which applies to functionals of type (1.1) when the integrand is singular (see Corollary 4.2). A typical example of a such singular integrand is given by $W: \mathbb{R}^{N} \times \mathbb{M}^{N \times N} \rightarrow[0,+\infty]$ of the form

$$
W(x, \xi)=|\xi|^{p}+a(x) h(\operatorname{det} \xi)
$$

where $0<\eta \leq a \in L^{\infty}\left(\mathbb{R}^{N}\right) \cap C\left(\mathbb{R}^{N}\right)$ is a 1-periodic function and $h: \mathbb{R} \rightarrow[0,+\infty]$ is a measurable function for which there exist $\gamma, \delta>0$ such that $h(t) \leq \delta$ for all $|t| \geq \gamma$. For example, given $s>0$ and $T \geq 0$ (possibly very large), this latter condition is satisfied with $\gamma=2 T$ and $\delta=\max \left\{\frac{1}{(2 T)^{s}}, T\right\}$ when $h$ is of type

$$
h(t)=\left\{\begin{array}{cl}
T & \text { if } t<-T \\
+\infty & \text { if } t \in[-T, 0] \\
\frac{1}{t^{s}} & \text { if } t>0 .
\end{array}\right.
$$

Note that $W$ as in (1.7) with $h$ given by (1.8) is compatible with the singular behavior $W(x, \xi) \rightarrow+\infty$ as $\operatorname{det} \xi \rightarrow 0$ (however, such a $W$ is not consistent with the noninterpenetration of the matter).

An outline of the paper is as follows. Our homogenization theorem (see Theorem 3.4 ) is stated and proved in $\S 3$. Its proof uses a relaxation theorem (see Theorem 2.7 ), whose statement and proof are given in $\S 2$, and Braides's homogenization theorem (see Theorem 3.2). Homogenization of functionals of type (1.1) when $W$ is of the form (1.7)-(1.8) is treated in $\S 4$ as an application of Theorem 3.4 (see Corollary 4.2).

\section{Relaxation THEOREM}

Let $m, N \geq 1$ be two integers. Given any bounded open set $D \subset \mathbb{R}^{N}$ with $|\partial D|=0$, we denote the space of continuous piecewise affine functions from $D$ to $\mathbb{R}^{m}$ by $\operatorname{Aff}\left(D ; \mathbb{R}^{m}\right)$, i.e., $\varphi \in \operatorname{Aff}\left(D ; \mathbb{R}^{m}\right)$ if and only if $\varphi$ is continuous and there exists a finite family $\left\{D_{i}\right\}_{i \in I}$ of open disjoint subsets of $D$ such that $\left|\partial D_{i}\right|=0$ for all $i \in I$, $\left|D \backslash \cup_{i \in I} D_{i}\right|=0$ and for every $i \in I, \nabla \varphi \equiv \xi_{i}$ in $D_{i}$ with $\xi_{i} \in \mathbb{M}^{m \times N}$, and we set $\operatorname{Aff}_{0}\left(D ; \mathbb{R}^{m}\right):=\left\{\varphi \in \operatorname{Aff}\left(D ; \mathbb{R}^{m}\right): \varphi=0\right.$ on $\left.\partial D\right\}$ and $W_{0}^{1, \infty}\left(D ; \mathbb{R}^{m}\right):=\{\varphi \in$ $W^{1, \infty}\left(D ; \mathbb{R}^{m}\right): \varphi=0$ on $\left.\partial D\right\}$. Given a normal integrand $f: \mathbb{R}^{N} \times \mathbb{M}^{m \times N} \rightarrow$ $[0,+\infty]$, where $\mathbb{M}^{m \times N}$ denotes the space of real $m \times N$ matrices, we consider the normal integrand $\mathcal{Z} f: \mathbb{R}^{N} \times \mathbb{M}^{m \times N} \rightarrow[0,+\infty]$ defined by

$$
\mathcal{Z} f(x, \xi):=\inf \left\{\int_{Y} f(x, \xi+\nabla \varphi(y)) d y: \varphi \in W_{0}^{1, \infty}\left(Y ; \mathbb{R}^{m}\right)\right\}
$$

with $Y:=] 0,1\left[^{N}\right.$. The following result is due to Fonseca (see [Fon88, lemma 2.16, Theorem 2.17 and Proposition 2.3]).

Proposition 2.1. The function $\mathcal{Z} f$ satisfies the following properties. 
(a) For every bounded open set $D \subset \mathbb{R}^{N}$ with $|\partial D|=0$ and every $(x, \xi) \in$ $\mathbb{R}^{N} \times \mathbb{M}^{m \times N}$

$$
\mathcal{Z} f(x, \xi)=\inf \left\{\frac{1}{|D|} \int_{D} f(x, \xi+\nabla \varphi(y)) d y: \varphi \in W_{0}^{1, \infty}\left(D ; \mathbb{R}^{m}\right)\right\} .
$$

(b) For every $x \in \mathbb{R}^{N}$, if $\mathcal{Z} f(x, \cdot)$ is finite then $\mathcal{Z} f(x, \cdot)$ is rank-one convex, i.e., for every $\xi, \xi^{\prime} \in \mathbb{M}^{m \times N}$ with $\operatorname{rank}\left(\xi-\xi^{\prime}\right) \leq 1$,

$$
\mathcal{Z} f\left(x, \lambda \xi+(1-\lambda) \xi^{\prime}\right) \leq \lambda \mathcal{Z} f(x, \xi)+(1-\lambda) \mathcal{Z} f\left(x, \xi^{\prime}\right) .
$$

(c) For every $x \in \mathbb{R}^{N}$, if $\mathcal{Z} f(x, \cdot)$ is finite then $\mathcal{Z} f(x, \cdot)$ is continuous, i.e., $\mathcal{Z} f$ is a Carathéodory integrand ${ }^{1}$ whenever $\mathcal{Z} f$ is finite.

(d) For every bounded open set $D \subset \mathbb{R}^{N}$ with $|\partial D|=0$, every $(x, \xi) \in \mathbb{R}^{N} \times$ $\mathbb{M}^{m \times N}$ and every $\varphi \in \operatorname{Aff}_{0}\left(D ; \mathbb{R}^{m}\right)$,

$$
\mathcal{Z} f(x, \xi) \leq \frac{1}{|D|} \int_{D} \mathcal{Z} f(x, \xi+\nabla \varphi(y)) d y .
$$

Remark 2.2. Proposition 2.1 is also valid with " $\hat{\mathcal{Z}} f$ " instead of " $\mathcal{Z} f$ " (see [AHM09, Proposition 2.3]) where $\hat{\mathcal{Z}} f: \mathbb{R}^{N} \times \mathbb{M}^{m \times N} \rightarrow[0,+\infty]$ is given by

$$
\hat{\mathcal{Z}} f(x, \xi):=\inf \left\{\int_{Y} f(x, \xi+\nabla \varphi(y)) d y: \varphi \in \operatorname{Aff}_{0}\left(Y ; \mathbb{R}^{m}\right)\right\} .
$$

In particular, Proposition 2.1(d) can be rewritten as $\hat{\mathcal{Z}}[\mathcal{Z} f]=\mathcal{Z} f$.

Given $x \in \mathbb{R}^{N}$ we say that $f(x, \cdot)$ is quasiconvex (in the sense of Morrey [Mor52]) if for every $\xi \in \mathbb{M}^{m \times N}$, every bounded open set $D \subset \mathbb{R}^{N}$ with $|\partial D|=0$ and every $\varphi \in W_{0}^{1, \infty}\left(D ; \mathbb{R}^{m}\right)$

$$
f(x, \xi) \leq \frac{1}{|D|} \int_{D} f(x, \xi+\nabla \varphi(y)) d y .
$$

By the quasiconvex envelope of $f(x, \cdot)$, that we denote by $\mathcal{Q} f(x, \cdot)$, we mean the greatest quasiconvex function which less than or equal to $f(x, \cdot)$. (clearly, $f(x, \cdot)$ is quasiconvex if and only if $\mathcal{Q} f(x, \cdot)=f(x, \cdot)$.) The concept of quasiconvex envelope was introduced by Dacorogna (see [Dac82]) who proved the following theorem (see [Dac08, Theorem 6.9 p. 271]).

Theorem 2.3. If $f$ is finite then $\mathcal{Q} f=\hat{\mathcal{Z}} f=\mathcal{Z} f$.

The following result is a slight generalization of Theorem 2.3.

Theorem 2.3-bis. If $\mathcal{Z} f$ is finite then $\mathcal{Q} f=\mathcal{Z} f$. In particular, $\mathcal{Z} f(x, \cdot)$ is quasiconvex for all $x \in \mathbb{R}^{N}$.

Proof. As $\mathcal{Z} f$ is finite we have $\mathcal{Q}[\mathcal{Z} f]=\hat{\mathcal{Z}}[\mathcal{Z} f]$ by Theorem 2.3. But $\hat{\mathcal{Z}}[\mathcal{Z} f]=\mathcal{Z} f$ from Remark 2.2 and so $\mathcal{Q}[\mathcal{Z} f]=\mathcal{Z} f$, i.e., $\mathcal{Z} f(x, \cdot)$ is quasiconvex for all $x \in \mathbb{R}^{N}$. As $\mathcal{Z} f \geq \mathcal{Q} f$ it follows that $\mathcal{Q} f=\mathcal{Z} f$.

Remark 2.4. Theorem 2.3 can be also generalized as follows: if $\hat{\mathcal{Z}} f$ is finite then $\mathcal{Q} f=\hat{\mathcal{Z}} f=\mathcal{Z} f$ (see [AHM09, Corollaire 2.17]).

From now on we fix $p>1$. Given $U \subset \mathbb{R}^{N}$ be a bounded open set with $|\partial U|=0$ we define $F: W^{1, p}\left(U ; \mathbb{R}^{m}\right) \rightarrow[0,+\infty]$ by

$$
F(\phi):=\int_{U} f(x, \nabla \phi(x)) d x
$$

and we consider the relaxed functionals $\bar{F}, \bar{F}_{0}: W^{1, p}\left(U ; \mathbb{R}^{m}\right) \rightarrow[0,+\infty]$ given by:

\footnotetext{
${ }^{1}$ A function $f: \mathbb{R}^{N} \times \mathbb{M}^{m \times N} \rightarrow[0,+\infty]$ is called a Carathéodory integrand if $f(x, \xi)$ is measurable in $x$ and continuous in $\xi$.
} 


$$
\begin{aligned}
& \diamond \bar{F}(\phi):=\inf \left\{\liminf _{n \rightarrow+\infty} F\left(\phi_{n}\right): \phi_{n} \rightarrow \phi \text { in } L^{p}\left(U ; \mathbb{R}^{m}\right)\right\} ; \\
& \diamond \bar{F}_{0}(\phi):=\inf \left\{\liminf _{n \rightarrow+\infty} F\left(\phi_{n}\right): W_{0}^{1, p}\left(U ; \mathbb{R}^{m}\right) \ni \phi_{n} \rightarrow \phi \text { in } L^{p}\left(U ; \mathbb{R}^{m}\right)\right\}
\end{aligned}
$$

with $W_{0}^{1, p}\left(U ; \mathbb{R}^{m}\right):=\left\{\phi \in W^{1, p}\left(U ; \mathbb{R}^{m}\right): \phi=0\right.$ on $\left.\partial U\right\}$. As $\bar{F}$ and $\bar{F}_{0}$ are not given by explicit formulas, it is of interest to know under which conditions on $f$ we have:

$$
\begin{aligned}
& \bar{F}(\phi)=\int_{U} \bar{f}(x, \nabla \phi(x)) d x \text { for all } \phi \in W^{1, p}\left(U ; \mathbb{R}^{m}\right) ; \\
& \bar{F}_{0}(\phi)=\left\{\begin{array}{cl}
\bar{F}(\phi) & \text { if } \phi \in W_{0}^{1, p}\left(U ; \mathbb{R}^{m}\right) \\
+\infty & \text { otherwise }
\end{array}\right.
\end{aligned}
$$

with $\bar{f}: \mathbb{R}^{N} \times \mathbb{M}^{m \times N} \rightarrow[0,+\infty]$ (whose we wish to give a representation formula). In the $p$-polynomial growth case, such integral representation problems was studied by Dacorogna (see [Dac82, Theorem 5], see also [Dac08, Theorem 9.1 p. 416]) and Acerbi and Fusco (see [AF84, Statement III.7]) who proved the following theorem.

Theorem 2.5. Let $f: \mathbb{R}^{N} \times \mathbb{M}^{m \times N} \rightarrow[0,+\infty]$ be a p-coercive Carathéodory integrand. If $f$ is of p-polynomial growth, i.e.,

$$
f(x, \xi) \leq c\left(1+|\xi|^{p}\right) \text { for all }(x, \xi) \in \mathbb{R}^{N} \times \mathbb{M}^{m \times N} \text { and some } c>0,
$$

then (2.1) and (2.2) hold with $\bar{f}=\mathcal{Z} f=\mathcal{Q} f$. If moreover $f(x,$.$) is quasiconvex$ for all $x \in \mathbb{R}^{N}$ then $\bar{f}=f$.

Because of the assumption (2.3) Acerbi-Dacorogna-Fusco's relaxation theorem cannot handle integrands having a singular behavior of type $f(x, \xi) \rightarrow+\infty$ as $\operatorname{det} \xi \rightarrow 0$ (when $m=N$ ). However, by using Theorem 2.5 and a key lemma (see Lemma 2.8) we can go beyond the $p$-polynomial growth case (see Theorem 2.7 and Corollary 4.4).

Let $\mathcal{A}^{p}$ be the class of $p$-coercive normal integrands $f: \mathbb{R}^{N} \times \mathbb{M}^{m \times N} \rightarrow[0,+\infty]$ satisfying the following two conditions:

$\left(\mathrm{C}_{1}\right)$ there exists a function $\omega:[0,+\infty[\rightarrow[0,+\infty[$ continuous at the origin with $\omega(0)=0$ such that for every $x_{1}, x_{2} \in \mathbb{R}^{N}$ and every $\xi \in \mathbb{M}^{m \times N}$,

$$
f\left(x_{1}, \xi\right) \leq \omega\left(\left|x_{1}-x_{2}\right|\right)\left(1+f\left(x_{2}, \xi\right)\right)+f\left(x_{2}, \xi\right) ;
$$

$\left(\mathrm{C}_{2}\right)$ the function $\mathcal{Z} f$ is of $p$-polynomial growth, i.e., $\mathcal{Z} f(x, \xi) \leq c\left(1+|\xi|^{p}\right)$ for all $(x, \xi) \in \mathbb{R}^{N} \times \mathbb{M}^{m \times N}$ and some $c>0$.

Remark 2.6. (i) Condition $\left(\mathrm{C}_{1}\right)$ is a condition of Serrin type (see [Ser61] or [Mor66, p. 96-97], see also [Mar86]).

(ii) If $f$ satisfies $\left(\mathrm{C}_{1}\right)$ then $\operatorname{dom} f\left(x_{1}, \cdot\right)=\operatorname{dom} f\left(x_{2}, \cdot\right)$ for all $x_{1}, x_{2} \in \mathbb{R}^{N}$, where, for $x \in \mathbb{R}^{N}, \operatorname{dom} f(x, \cdot):=\left\{\xi \in \mathbb{M}^{m \times N}: f(x, \xi)<+\infty\right\}$.

(iii) If $f$ satisfies $\left(\mathrm{C}_{1}\right)$ then $f(\cdot, \xi)$ is continuous for all $\xi \in \mathbb{M}^{m \times N}$.

(iv) If $f$ satisfies $\left(C_{1}\right)$ then for every $x_{1}, x_{2} \in \mathbb{R}^{N}$ and every $\xi \in \mathbb{M}^{m \times N}$,

$$
\mathcal{Z} f\left(x_{1}, \xi\right) \leq \omega\left(\left|x_{1}-x_{2}\right|\right)\left(1+\mathcal{Z} f\left(x_{2}, \xi\right)\right)+\mathcal{Z} f\left(x_{2}, \xi\right) .
$$

In particular, we have $\mathcal{Z} f(\cdot, \xi) \in C(\bar{V})$ for all $\xi \in \mathbb{M}^{m \times N}$ whenever $f$ satisfies $\left(\mathrm{C}_{1}\right), \mathcal{Z} f$ is finite (for example when $\left(\mathrm{C}_{2}\right)$ holds) and $V \subset \mathbb{R}^{N}$ is a bounded open set. (This fact will be used in the proof of Lemma 2.8.)

The following theorem is a slight generalization of [AHM07, Theorem 2] (see also [AHM08, Theorem 1.4] and [AHM09, Théorème 2.1]). 
Theorem 2.7. If $f \in \mathcal{A}^{p}$ then (2.1) and (2.2) hold with $\bar{f}=\mathcal{Z} f=\mathcal{Q} f$. As a consequence, we have

$$
\inf _{\phi \in W_{0}^{1, p}\left(U ; \mathbb{R}^{m}\right)} \int_{U} f(x, \nabla \phi(x)) d x=\inf _{\phi \in W_{0}^{1, p}\left(U ; \mathbb{R}^{m}\right)} \int_{U} \mathcal{Z} f(x, \nabla \phi(x)) d x .
$$

Proof. Let $\mathcal{Z} F: W^{1, p}\left(U ; \mathbb{R}^{m}\right) \rightarrow[0,+\infty]$ be defined by

$$
\mathcal{Z} F(\phi):=\int_{U} \mathcal{Z} f(x, \nabla \phi(x)) d x
$$

and let $\overline{\mathcal{Z F}}, \overline{\mathcal{Z F}}_{0}: W^{1, p}\left(U ; \mathbb{R}^{m}\right) \rightarrow[0,+\infty]$ be given by:

$$
\begin{aligned}
& \diamond \overline{\mathcal{Z F}}(\phi):=\inf \left\{\liminf _{n \rightarrow+\infty} \mathcal{Z} F\left(\phi_{n}\right): \phi_{n} \rightarrow \phi \text { in } L^{p}\left(U ; \mathbb{R}^{m}\right)\right\} ; \\
& \diamond \overline{\mathcal{Z F}}_{0}(\phi):=\inf \left\{\liminf _{n \rightarrow+\infty} \mathcal{Z} F\left(\phi_{n}\right): W_{0}^{1, p}\left(U ; \mathbb{R}^{m}\right) \ni \phi_{n} \rightarrow \phi \text { in } L^{p}\left(U ; \mathbb{R}^{m}\right)\right\} .
\end{aligned}
$$

We need the following lemma whose proof is given below.

Lemma 2.8. Under $\left(\mathrm{C}_{1}\right)$ and $\left(\mathrm{C}_{2}\right)$ if $\phi \in \operatorname{Aff}\left(U ; \mathbb{R}^{m}\right)\left(\operatorname{resp} . \phi \in \operatorname{Aff}_{0}\left(U ; \mathbb{R}^{m}\right)\right)$ then

$$
\bar{F}(\phi) \leq \int_{U} \mathcal{Z} f(x, \nabla \phi(x)) d x\left(\operatorname{resp} . \bar{F}_{0}(\phi) \leq \int_{U} \mathcal{Z} f(x, \nabla \phi(x)) d x\right) .
$$

As $\mathcal{Z} f$ is of $p$-polynomial growth and $\operatorname{Aff}\left(U ; \mathbb{R}^{m}\right)$ (resp. $\left.\operatorname{Aff}_{0}\left(U ; \mathbb{R}^{m}\right)\right)$ is strongly dense in $W^{1, p}\left(U ; \mathbb{R}^{m}\right)$ (resp. $\left.W_{0}^{1, p}\left(U ; \mathbb{R}^{m}\right)\right)$, from Lemma 2.8 we deduce that $(2.4)$ holds for all $\phi \in W^{1, p}\left(U ; \mathbb{R}^{m}\right)$ (resp. $\phi \in W_{0}^{1, p}\left(U ; \mathbb{R}^{m}\right)$ ). Thus $\bar{F} \leq \overline{\mathcal{Z} F}$ (resp. $\bar{F}_{0} \leq \overline{\mathcal{Z} F}_{0}$ ). Moreover, $\overline{\mathcal{Z} F} \leq \bar{F}$ (resp. $\overline{\mathcal{Z} F}_{0} \leq \bar{F}_{0}$ ), hence

$$
\bar{F}=\overline{\mathcal{Z} F}\left(\text { resp. } \bar{F}_{0}=\overline{\mathcal{Z F}}_{0}\right) \text {. }
$$

As $f$ is $p$-coercive, also is $\mathcal{Z} f$. Moreover, since $\mathcal{Z} f$ is finite (because $\left(\mathrm{C}_{2}\right)$ holds), on the one hand, $\mathcal{Z} f$ is a Carathéodory integrand by Proposition 2.1(c) and, on the other hand, $\mathcal{Z} f(x, \cdot)$ is quasiconvex for all $x \in \mathbb{R}^{N}$ by Theorem 2.3-bis. From Acerbi-Dacorogna-Fusco's relaxation theorem (see Theorem 2.5) it follows that

$$
\overline{\mathcal{Z} F}=\mathcal{Z} F\left(\operatorname{resp} . \overline{\mathcal{Z F}}_{0}=\left\{\begin{array}{ll}
\mathcal{Z} F & \text { on } W_{0}^{1, p}\left(U ; \mathbb{R}^{m}\right) \\
+\infty & \text { elsewhere }
\end{array}\right)\right.
$$

which gives the theorem when combined with (2.5).

Proof of Lemma 2.8. By definition, there exists a finite family $\left\{U_{i}\right\}_{i \in I}$ of open disjoint subsets of $U$ such that $\left|\partial U_{i}\right|=0$ for all $i \in I,\left|U \backslash \cup_{i \in I} U_{i}\right|=0$ and, for every $i \in I, \nabla \phi \equiv \xi_{i}$ in $U_{i}$ with $\xi_{i} \in \mathbb{M}^{m \times N}$. Thus

$$
\int_{U} \mathcal{Z} f(x, \nabla \phi(x)) d x=\sum_{i \in I} \int_{U_{i}} \mathcal{Z} f\left(x, \xi_{i}\right) d x .
$$

From Remark 2.6(iv) we see that $\mathcal{Z} f\left(\cdot, \xi_{i}\right) \in C\left(\bar{U}_{i}\right)$ for all $i \in I$. Hence, for each $i \in I$, there exists a finite family $\left\{U_{i, j}^{k}\right\}_{j \in J_{i}^{k}}$ of disjoint subsets of $U_{i}$ with $\left|\partial U_{i, j}^{k}\right|=0$ for all $j \in J_{i}^{k}$ and $\left|U_{i} \backslash \cup_{j \in J_{i}^{k}} U_{i, j}^{k}\right|=0$ such that:

$$
\begin{aligned}
& \operatorname{diam}\left(U_{i, j}^{k}\right)<\frac{1}{k} \text { for all } j \in J_{i}^{k} \\
& \lim _{k \rightarrow+\infty} \sum_{j \in J_{i}^{k}}\left|U_{i, j}^{k}\right| \mathcal{Z} f\left(x_{i, j}^{k}, \xi_{i}\right)=\int_{U_{i}} \mathcal{Z} f\left(x, \xi_{i}\right) d x,
\end{aligned}
$$

where, for $X \subset \mathbb{R}^{N}, \operatorname{diam}(X):=\sup \left\{\left|x_{1}-x_{2}\right|: x_{1}, x_{2} \in X\right\}$. Fix any $\delta>0$. Then, there exists $\eta>0$ such that

$$
\omega(t)<\delta \text { for all }|t|<\eta
$$


where the function $\omega:[0,+\infty[\rightarrow[0,+\infty[$ (continuous at the origin with $\omega(0)=0)$ are given by $\left(\mathrm{C}_{1}\right)$. Fix any $k \geq 1$ such that $\frac{1}{k}<\eta$. Given any $i \in I$ and any $j \in J_{i}^{k}$ we consider $\varphi_{i, j} \in W_{0}^{1, \infty}\left(Y ; \mathbb{R}^{m}\right)$ such that

$$
\int_{Y} f\left(x_{i, j}^{k}, \xi_{i}+\nabla \varphi_{i, j}(y)\right) d y \leq \mathcal{Z} f\left(x_{i, j}^{k}, \xi_{i}\right)+\frac{\delta}{|U|} .
$$

By Vitali's covering theorem, there exists a finite or countable family $\left\{a_{i, j, \ell}+\right.$ $\left.\alpha_{i, j, \ell} Y\right\}_{\ell \in L_{i, j}}$ of disjoint subsets of $U_{i, j}^{k}$, where $a_{i, j, \ell} \in \mathbb{R}^{N}$ and $0<\alpha_{i, j, \ell}<\frac{1}{n}$, such that $\left|U_{i, j}^{k} \backslash \cup_{\ell \in L_{i, j}}\left(a_{i, j, \ell}+\alpha_{i, j, \ell} Y\right)\right|=0$ (and so $\left.\sum_{\ell \in L_{i, j}} \alpha_{i, j, \ell}^{N}=\left|U_{i, j}^{k}\right|\right)$. Define $\phi_{n} \in W_{0}^{1, p}\left(U ; \mathbb{R}^{m}\right)$ by

$$
\phi_{n}(x):=\alpha_{i, j, \ell} \varphi_{i, j}\left(\frac{x-a_{i, j, \ell}}{\alpha_{i, j, \ell}}\right) \text { if } x \in a_{i, j, \ell}+\alpha_{i, j, \ell} Y .
$$

Then:

$\diamond\left\|\phi_{n}\right\|_{L^{\infty}\left(U ; \mathbb{R}^{m}\right)} \leq \frac{1}{n} \max _{i \in I, j \in J_{i}^{k}}\left\|\varphi_{i, j}\right\|_{L^{\infty}\left(Y ; \mathbb{R}^{m}\right)}$ for all $n \geq 1 ;$

$\diamond\left\|\nabla \phi_{n}\right\|_{L^{\infty}\left(U ; \mathbb{R}^{m}\right)} \leq \max _{i \in I, j \in J_{i}^{k}}\left\|\nabla \varphi_{i, j}\right\|_{L^{\infty}\left(Y ; \mathbb{R}^{m}\right)}$ for all $n \geq 1$,

hence (up to a subsequence) $\phi_{n} \stackrel{*}{\rightarrow} 0$ in $W^{1, \infty}\left(U ; \mathbb{R}^{m}\right)$, where "**" denotes the weak* convergence in $W^{1, \infty}\left(U ; \mathbb{R}^{m}\right)$. Consequently $\phi_{n} \rightarrow 0$ in $W^{1, p}\left(U ; \mathbb{R}^{m}\right)$ and so (up to a subsequence) $\phi_{n} \rightarrow 0$ in $L^{p}\left(U ; \mathbb{R}^{m}\right)$. Using $(2.9)$ and $\left(\mathrm{C}_{2}\right)$ we see that

$$
\sum_{i \in I} \sum_{j \in J_{i}^{k}} \int_{U_{i, j}^{k}} f\left(x_{i, j}^{k}, \xi_{i}+\nabla \phi_{n}(x)\right) d x \leq \frac{c}{C}\left(|U|+\|\nabla \phi\|_{L^{p}\left(U ; \mathbb{R}^{m}\right)}^{p}\right)+\frac{\delta}{C} \text { for all } n \geq 1
$$

with $c, C>0$. Taking $\left(\mathrm{C}_{1}\right),(2.6)$ and (2.8) into account it follows that for every $n \geq 1$,

$$
\int_{U} f\left(x, \nabla \phi(x)+\nabla \phi_{n}(x)\right) d x \leq K(\delta)+\sum_{i \in I} \sum_{j \in J_{i}^{k}} \int_{U_{i, j}^{k}} f\left(x_{i, j}^{k}, \xi_{i}+\nabla \phi_{n}(x)\right) d x
$$

with $\delta\left(|U|+\frac{c}{C}\left(|U|+\|\nabla \phi\|_{L^{p}\left(U ; \mathbb{R}^{m}\right)}^{p}\right)+\frac{\delta}{C}\right)=: K(\delta) \rightarrow 0$ as $\delta \rightarrow 0$. Moreover, from (2.9) we have

$$
\begin{aligned}
\sum_{i \in I} \sum_{j \in J_{i}^{k}} \int_{U_{i, j}^{k}} f\left(x_{i, j}^{k}, \xi_{i}+\nabla \phi_{n}(x)\right) d x & =\sum_{i \in I} \sum_{j \in J_{i}^{k}}\left|U_{i, j}^{k}\right| \int_{Y} f\left(x_{i, j}^{k}, \xi_{i}+\nabla \varphi_{i, j}(x)\right) d x \\
& \leq \sum_{i \in I} \sum_{j \in J_{i}^{k}}\left|U_{i, j}^{k}\right| \mathcal{Z} f\left(x_{i, j}^{k}, \xi_{i}\right)+\delta
\end{aligned}
$$

hence, for every $n \geq 1$,

$$
\int_{U} f\left(x, \nabla \phi(x)+\nabla \phi_{n}(x)\right) d x \leq \sum_{i \in I} \sum_{j \in J_{i}^{k}}\left|U_{i, j}^{k}\right| \mathcal{Z} f\left(x_{i, j}^{k}, \xi_{i}\right)+K(\delta)+\delta .
$$

As $\phi+\phi_{n} \in W^{1, p}\left(U ; \mathbb{R}^{m}\right)\left(\right.$ resp. $\left.\phi+\phi_{n} \in W_{0}^{1, p}\left(U ; \mathbb{R}^{m}\right)\right)$ for all $n \geq 1$ and $\phi+\phi_{n} \rightarrow \phi$ in $L^{p}\left(U ; \mathbb{R}^{m}\right)$ it follows that

$$
\begin{gathered}
\bar{F}(\phi) \leq \sum_{i \in I} \sum_{j \in J_{i}^{k}}\left|U_{i, j}^{k}\right| \mathcal{Z} f\left(x_{i, j}^{k}, \xi_{i}\right)+K(\delta)+\delta \\
\text { (resp. } \left.\bar{F}_{0}(\phi) \leq \sum_{i \in I} \sum_{j \in J_{i}^{k}}\left|U_{i, j}^{k}\right| \mathcal{Z} f\left(x_{i, j}^{k}, \xi_{i}\right)+K(\delta)+\delta\right) .
\end{gathered}
$$

Letting $k \rightarrow+\infty$ and using (2.7) we deduce that

$$
\bar{F}(\phi) \leq \int_{U} \mathcal{Z} f(x, \nabla \phi(x)) d x+K(\delta)+\delta
$$




$$
\text { (resp. } \left.\bar{F}_{0}(\phi) \leq \int_{U} \mathcal{Z} f(x, \nabla \phi(x)) d x+K(\delta)+\delta\right)
$$

and (2.4) follows by letting $\delta \rightarrow 0$.

\section{Homogenization THEOREM}

Let $\Omega \subset \mathbb{R}^{N}$ be a bounded open set with $|\partial \Omega|=0$, let $W: \mathbb{R}^{N} \times \mathbb{M}^{m \times N} \rightarrow[0,+\infty]$ be a normal integrand and, for each $\varepsilon>0$, let $I_{\varepsilon}: W^{1, p}\left(\Omega ; \mathbb{R}^{m}\right) \rightarrow[0,+\infty]$ (with $p>1$ ) be defined by (1.1). To accomplish our asymptotic analysis as $\varepsilon \rightarrow 0$, we will use De Giorgi's $\Gamma$-convergence which can be defined as follows (for more details see [DM93, BD98, Bra06]).

Definition 3.1. We say that $I_{\varepsilon} \Gamma$-converges to $I_{\text {hom }}: W^{1, p}\left(\Omega ; \mathbb{R}^{m}\right) \rightarrow[0,+\infty]$ with respect to the $L^{p}\left(\Omega ; \mathbb{R}^{m}\right)$-convergence as $\varepsilon \rightarrow 0$, and we write $I_{\text {hom }}=\Gamma$ - $\lim _{\varepsilon \rightarrow 0} I_{\varepsilon}$, if

$$
\left(\Gamma-\liminf _{\varepsilon \rightarrow 0} I_{\varepsilon}\right)(\phi)=\left(\Gamma-\limsup _{\varepsilon \rightarrow 0} I_{\varepsilon}\right)(\phi)=I_{\mathrm{hom}}(\phi)
$$

for all $\phi \in W^{1, p}\left(\Omega ; \mathbb{R}^{m}\right)$ with:

$$
\begin{gathered}
\left(\Gamma-\liminf _{\varepsilon \rightarrow 0} I_{\varepsilon}\right)(\phi):=\inf \left\{\liminf _{\varepsilon \rightarrow 0} I_{\varepsilon}\left(\phi_{\varepsilon}\right): \phi_{\varepsilon} \rightarrow \phi \text { in } L^{p}\left(\Omega ; \mathbb{R}^{m}\right)\right\} ; \\
\left(\Gamma-\limsup _{\varepsilon \rightarrow 0} I_{\varepsilon}\right)(\phi):=\inf \left\{\limsup _{\varepsilon \rightarrow 0} I_{\varepsilon}\left(\phi_{\varepsilon}\right): \phi_{\varepsilon} \rightarrow \phi \text { in } L^{p}\left(\Omega ; \mathbb{R}^{m}\right)\right\} .
\end{gathered}
$$

Braides proved, in the $p$-polynomial growth case, the following theorem (see [Bra85, BD98], see also [Mül87]).

Theorem 3.2. If $W$ satisfies (1.2), (1.3) and (1.4) then $I_{\mathrm{hom}}=\Gamma-\lim _{\varepsilon \rightarrow 0} I_{\varepsilon}$ with $I_{\text {hom }}$ defined by $(1.5)$ and $W_{\text {hom }}: \mathbb{M}^{m \times N} \rightarrow[0,+\infty]$ given by (1.6).

To prove our homogenization theorem (see Theorem 3.4) we will need Theorems 2.7 and 3.2 and the following classical property of the $\Gamma$-convergence (see [Bra06, Proposition 2.5]).

Proposition 3.3. The $\Gamma$-limit is stable by substituting $I_{\varepsilon}$ by its relaxed functional $\bar{I}_{\varepsilon}$, i.e.,

$$
\Gamma-\liminf _{\varepsilon \rightarrow 0} I_{\varepsilon}=\Gamma-\liminf _{\varepsilon \rightarrow 0} \bar{I}_{\varepsilon} \text { and } \Gamma-\limsup _{\varepsilon \rightarrow 0} I_{\varepsilon}=\Gamma-\limsup _{\varepsilon \rightarrow 0} \bar{I}_{\varepsilon},
$$

where, for each $\varepsilon>0, \bar{I}_{\varepsilon}: W^{1, p}\left(\Omega ; \mathbb{R}^{m}\right) \rightarrow[0,+\infty]$ is given by

$$
\bar{I}_{\varepsilon}(\phi):=\inf \left\{\liminf _{n \rightarrow+\infty} I_{\varepsilon}\left(\phi_{n}\right): \phi_{n} \rightarrow \phi \text { in } L^{p}\left(\Omega ; \mathbb{R}^{m}\right)\right\} .
$$

Set $\mathcal{A}_{\text {per }}^{p}:=\left\{f \in \mathcal{A}^{p}: f\right.$ is 1-periodic $\}$. The main result of the paper is the following. (When $m=N$, it can handle integrands having a singular behavior of type $W(x, \xi) \rightarrow+\infty$ as $\operatorname{det} \xi \rightarrow 0$, see Corollary 4.2 ).

Theorem 3.4. If $W \in \mathcal{A}_{\mathrm{per}}^{p}$ then $I_{\mathrm{hom}}=\Gamma-\lim _{\varepsilon \rightarrow 0} I_{\varepsilon}$ with $I_{\mathrm{hom}}$ defined by (1.5) and $W_{\text {hom }}: \mathbb{M}^{m \times N} \rightarrow[0,+\infty]$ given by (1.6).

Proof. By Proposition 3.3 it suffices to prove Theorem 3.4 with " $\bar{I}_{\varepsilon}$ " instead of " $I_{\varepsilon}$ ". Fix any $\varepsilon>0$ and consider $f_{\varepsilon}: \mathbb{R}^{N} \times \mathbb{M}^{m \times N} \rightarrow[0,+\infty]$ given by $f_{\varepsilon}(x, \xi):=$ $W\left(\frac{x}{\varepsilon}, \xi\right)$. As $W \in \mathcal{A}_{\text {per }}^{p}$ and $\mathcal{Z} f_{\varepsilon}(x, \xi)=\mathcal{Z} W\left(\frac{x}{\varepsilon}, \xi\right)$ for all $(x, \xi) \in \mathbb{R}^{N} \times \mathbb{M}^{m \times N}$ it is easy to see that $f_{\varepsilon} \in \mathcal{A}^{p}$. Applying Theorem 2.7 (with $f=f_{\varepsilon}$ ) we deduce that for every $\varepsilon>0$,

$$
\bar{I}_{\varepsilon}(\phi)=\int_{\Omega} \mathcal{Z} W\left(\frac{x}{\varepsilon}, \nabla \phi(x)\right) d x
$$


where $\mathcal{Z} W$ is clearly $p$-coercive, 1 -periodic and of $p$-polynomial growth. From Braides's homogenization theorem (see Theorem 3.2) it follows that $I_{\text {hom }}=\Gamma-\lim _{\varepsilon \rightarrow 0} \bar{I}_{\varepsilon}$ with $I_{\text {hom }}$ defined by $(1.5)$ and $W_{\text {hom }}: \mathbb{M}^{m \times N} \rightarrow[0,+\infty]$ given by

$$
W_{\text {hom }}(\xi)=\inf _{k \geq 1} \frac{1}{k^{N}} \inf \left\{\int_{k Y} \mathcal{Z} W(x, \xi+\nabla \varphi(x)) d x: \varphi \in W_{0}^{1, p}\left(k Y ; \mathbb{R}^{m}\right)\right\} .
$$

Fix any $k \geq 1$, any $\xi \in \mathbb{M}^{m \times N}$ and consider $f_{\xi}: \mathbb{R}^{N} \times \mathbb{M}^{m \times N} \rightarrow[0,+\infty]$ given by $f_{\xi}(x, \zeta):=W(x, \xi+\zeta)$. As $W \in \mathcal{A}_{\text {per }}^{p}$ and $\mathcal{Z} f_{\xi}(x, \zeta)=\mathcal{Z} W(x, \xi+\zeta)$ for all $(x, \zeta) \in \mathbb{R}^{N} \times \mathbb{M}^{m \times N}$ it is easy to see that $f_{\xi} \in \mathcal{A}^{p}$. Applying Theorem 2.7 (with $U=k Y$ and $f=f_{\xi}$ ) we deduce that for every $k \geq 1$ and every $\xi \in \mathbb{M}^{m \times N}$,

$$
\inf _{\varphi \in W_{0}^{1, p}\left(k Y ; \mathbb{R}^{m}\right)} \int_{k Y} W(x, \xi+\nabla \varphi(x)) d x=\inf _{\varphi \in W_{0}^{1, p}\left(k Y ; \mathbb{R}^{m}\right)} \int_{k Y} \mathcal{Z} W(x, \xi+\nabla \varphi(x)) d x
$$

and the theorem follows.

\section{Application}

The following condition on the normal integrand $f: \mathbb{R}^{N} \times \mathbb{M}^{N \times N} \rightarrow[0,+\infty]$ is compatible with a singular behavior of type $f(x, \xi) \rightarrow+\infty$ as $\operatorname{det} \xi \rightarrow 0$.

$\left(\hat{\mathrm{C}}_{2}\right)$ There exist $\alpha, \beta>0$ such that for every $(x, \xi) \in \mathbb{R}^{N} \times \mathbb{M}^{N \times N}$,

$$
\text { if }|\operatorname{det} \xi| \geq \alpha \text { then } f(x, \xi) \leq \beta\left(1+|\xi|^{p}\right) .
$$

Typically, the function $H: \mathbb{R}^{N} \times \mathbb{M}^{N \times N} \rightarrow[0,+\infty]$ defined by

$$
H(x, \xi):=|\xi|^{p}+a(x) h(\operatorname{det} \xi),
$$

where $0 \leq a \in L^{\infty}\left(\mathbb{R}^{N}\right)$ and $h: \mathbb{R} \rightarrow[0,+\infty[$ is a measurable function for which there exist $\gamma, \delta>0$ such that $h(t) \leq \delta$ for all $|t| \geq \gamma$, satisfies $\left(\hat{\mathrm{C}}_{2}\right)$ with $\alpha=\gamma$ and $\beta=\max \left\{1, \delta\|a\|_{L^{\infty}\left(\mathbb{R}^{N}\right)}\right\}$. The singular behavior $H(x, \xi) \rightarrow+\infty$ as $\operatorname{det} \xi \rightarrow 0$ is possible (for example when $h$ is given by (1.8)).

Denote the class of $p$-coercive normal integrands $f: \mathbb{R}^{N} \times \mathbb{M}^{N \times N} \rightarrow[0,+\infty]$ satisfying $\left(\mathrm{C}_{1}\right)$ and $\left(\hat{\mathrm{C}}_{2}\right)$ by $\mathcal{S}^{p}$ and set $\mathcal{S}_{\text {per }}^{p}:=\left\{f \in \mathcal{S}^{p}: f\right.$ is 1-periodic $\}$. When $0<\eta \leq a \in L^{\infty}\left(\mathbb{R}^{N}\right) \cap C\left(\mathbb{R}^{N}\right)$ we have $H \in \mathcal{S}^{p}$ since $H$ satisfies $\left(\mathrm{C}_{1}\right)$ with $\omega(t):=\frac{1}{\eta} \sup \left\{\left|a\left(x_{1}\right)-a\left(x_{2}\right)\right|:\left|x_{1}-x_{2}\right| \leq t\right\}$. If moreover $a$ is 1-periodic then $H \in \mathcal{S}_{\text {per }}^{p}$.

The following theorem, whose proof is given below, is a slight improvement of [AHM08, Proposition 1.8] (see also [AHM09, théorème 2.20]).

Theorem 4.1. Let $f: \mathbb{R}^{N} \times \mathbb{M}^{N \times N} \rightarrow[0,+\infty]$ be a normal integrand. If $f$ satisfies $\left(\hat{\mathrm{C}}_{2}\right)$ then $\mathcal{Z} f$ is of p-polynomial growth, i.e.,

$$
\mathcal{Z} f(x, \xi) \leq c\left(1+|\xi|^{p}\right) \text { for all }(x, \xi) \in \mathbb{R}^{N} \times \mathbb{M}^{N \times N} \text { and some } c>0 .
$$

By Theorem 4.1 we have $\mathcal{S}^{p} \subset \mathcal{A}^{p}$ and so $\mathcal{S}_{\text {per }}^{p} \subset \mathcal{A}_{\text {per }}^{p}$. Hence, as a direct consequence of Theorems 4.1 and 3.4 we have

Corollary 4.2. If $W \in \mathcal{S}_{\mathrm{per}}^{p}$ then $I_{\mathrm{hom}}=\Gamma-\lim _{\varepsilon \rightarrow 0} I_{\varepsilon}$ with $I_{\mathrm{hom}}$ defined by (1.5) and $W_{\text {hom }}: \mathbb{M}^{N \times N} \rightarrow[0,+\infty]$ given by (1.6).

Remark 4.3. From Theorems 4.1 and 2.7 we obtain

Corollary 4.4. If $f \in \mathcal{S}^{p}$ then (2.1) and (2.2) hold with $\bar{f}=\mathcal{Z} f=\mathcal{Q} f$.

Corollary 4.4 slightly improves [AHM09, Corollaire 2.22] (see also [AHM08, Theorem 1.3]). 
To prove Theorem 4.1 we need the following two lemmas. The first is a special case of a theorem due to Dacorogna and Ribeiro (see [DR04, Theorem 1.3], see also [Dac08, Theorem 10.29 p. 462]) and the second is a special case of a theorem due to Ben Belgacem (see [BB96], see also [AHM09, Théorème 3.25] for a proof).

Lemma 4.5. Given $t_{1}<t_{2}$ and $\xi \in \mathbb{M}^{N \times N}$ with $t_{1}<\operatorname{det} \xi<t_{2}$ there exists $\varphi \in W_{0}^{1, \infty}\left(Y ; \mathbb{R}^{N}\right)$ such that $\operatorname{det}(\xi+\nabla \varphi(y)) \in\left\{t_{1}, t_{2}\right\}$ for a.e. $y \in Y$.

Lemma 4.6. Let $f: \mathbb{R}^{N} \times \mathbb{M}^{N \times N} \rightarrow[0,+\infty]$ be a normal integrand. If $f$ satisfies $\left(\hat{\mathrm{C}}_{2}\right)$ then $\mathcal{R} f$ is of p-polynomial growth, where for every $x \in \mathbb{R}^{N}, \mathcal{R} f(x, \cdot)$ denotes the rank-one convex envelope of $f(x, \cdot)$, i.e., the greatest rank-one convex function which less than or equal to $f(x, \cdot)$.

Proof of Theorem 4.1. Fix any $x \in \mathbb{R}^{N}$ and any $\xi \in \mathbb{M}^{N \times N}$. Clearly, if $|\operatorname{det} \xi| \geq \alpha$ then $\mathcal{Z} f(x, \xi)<+\infty$. On the other hand, if $|\operatorname{det} \xi|<\alpha$ then, by Lemma 4.5 , there exists $\varphi \in W_{0}^{1, \infty}\left(Y ; \mathbb{R}^{N}\right)$ such that $\mid \operatorname{det}(\xi+\nabla \varphi(y) \mid=\alpha$ for a.e. $y \in Y$, and using $\left(\hat{\mathrm{C}}_{2}\right)$ we see that

$$
\mathcal{Z} f(x, \xi) \leq \int_{Y} f(x, \xi+\nabla \varphi(y)) d y \leq 2^{p} \beta\left(1+|\xi|^{p}+\|\nabla \varphi\|_{L^{p}\left(Y ; \mathbb{R}^{N}\right)}^{p}\right)<+\infty .
$$

Thus $\mathcal{Z} f(x, \xi)<+\infty$ for all $\xi \in \mathbb{M}^{N \times N}$, i.e., $\mathcal{Z} f(x, \cdot)$ is finite. From Proposition 2.1(b) we deduce that $\mathcal{Z} f(x, \cdot)$ is rank-one convex. Hence $\mathcal{Z} f(x, \cdot) \leq \mathcal{R} f(x, \cdot)$ for all $x \in \mathbb{R}^{N}$, i.e., $\mathcal{Z} f \leq \mathcal{R} f$, and the theorem follows from Lemma 4.6.

\section{REFERENCES}

[AF84] Emilio Acerbi and Nicola Fusco. Semicontinuity problems in the calculus of variations. Arch. Rational Mech. Anal., 86(2):125-145, 1984.

[AHM07] Omar Anza Hafsa and Jean-Philippe Mandallena. Relaxation of variational problems in two-dimensional nonlinear elasticity. Ann. Mat. Pura Appl. (4), 186(1):187-198, 2007.

[AHM08] Omar Anza Hafsa and Jean-Philippe Mandallena. Relaxation theorems in nonlinear elasticity. Ann. Inst. H. Poincaré Anal. Non Linéaire, 25(1):135-148, 2008.

[AHM09] Omar Anza Hafsa and Jean-Philippe Mandallena. Relaxation et passage 3d-2d avec contraintes de type déterminant. ArXiv:math.AP/0901.3688, 2009.

[BB96] Hafedh Ben Belgacem. Modélisation de structures minces en élasticité non linéaire. PhD thesis, Université Pierre et Marie Curie, 1996.

[BD98] Andrea Braides and Anneliese Defranceschi. Homogenization of multiple integrals, volume 12 of Oxford Lecture Series in Mathematics and its Applications. The Clarendon Press Oxford University Press, New York, 1998.

[Bra85] Andrea Braides. Homogenization of some almost periodic coercive functional. Rend. Accad. Naz. Sci. XL Mem. Mat. (5), 9(1):313-321, 1985.

[Bra06] Andrea Braides. A handbook of $\gamma$-convergence. In M. Chipot and P. Quittner, editors, Handbook of Differential equations. Stationary Partial Differential Equations, volume 3. Elsevier, 2006.

[CCDAG02] L. Carbone, D. Cioranescu, R. De Arcangelis, and A. Gaudiello. Homogenization of unbounded functionals and nonlinear elastomers. The general case. Asymptot. Anal., 29(3-4):221-272, 2002.

[CCDAG04] Luciano Carbone, Doina Cioranescu, Riccardo De Arcangelis, and Antonio Gaudiello. Homogenization of unbounded functionals and nonlinear elastomers. The case of the fixed constraints set. ESAIM Control Optim. Calc. Var., 10(1):53-83 (electronic), 2004.

[Dac82] B. Dacorogna. Quasiconvexity and relaxation of nonconvex problems in the calculus of variations. J. Funct. Anal., 46(1):102-118, 1982.

[Dac08] Bernard Dacorogna. Direct methods in the calculus of variations, volume 78 of $A p$ plied Mathematical Sciences. Springer, New York, second edition, 2008.

[DM93] Gianni Dal Maso. An introduction to $\Gamma$-convergence. Progress in Nonlinear Differential Equations and their Applications, 8. Birkhäuser Boston Inc., Boston, MA, 1993. 
[DR04] Bernard Dacorogna and Ana Margarida Ribeiro. Existence of solutions for some implicit partial differential equations and applications to variational integrals involving quasi-affine functions. Proc. Roy. Soc. Edinburgh Sect. A, 134(5):907-921, 2004.

[Fon88] Irene Fonseca. The lower quasiconvex envelope of the stored energy function for an elastic crystal. J. Math. Pures Appl. (9), 67(2):175-195, 1988.

[Mar86] Paolo Marcellini. On the definition and the lower semicontinuity of certain quasiconvex integrals. Ann. Inst. H. Poincaré Anal. Non Linéaire, 3(5):391-409, 1986.

[Mor52] Charles B. Morrey, Jr. Quasi-convexity and the lower semicontinuity of multiple integrals. Pacific J. Math., 2:25-53, 1952.

[Mor66] Charles B. Morrey, Jr. Multiple integrals in the calculus of variations. Die Grundlehren der mathematischen Wissenschaften, Band 130. Springer-Verlag New York, Inc., New York, 1966.

[Mül87] Stefan Müller. Homogenization of nonconvex integral functionals and cellular elastic materials. Arch. Rational Mech. Anal., 99(3):189-212, 1987.

[Ser61] James Serrin. On the definition and properties of certain variational integrals. Trans. Amer. Math. Soc., 101:139-167, 1961.

UNIVERSITE MONTPELliER II, UMR-CNRS 5508, LMGC, Place Eugìne Bataillon, 34095 Montpellier, France.

E-mail address: Omar.Anza-Hafsa@univ-montp2.fr

UNIVERSite DE MEDEA, LMP2M (Laboratoire de Mécanique, Physique et Modélisation Mathématique), Quartier Ain-D’Heb, MÉdéa (26000), Algerie.

E-mail address: leghmizi@hotmail.com

Universite de nimes, Site des Carmes, Place Gabriel Péri, 30021 Nîmes, France.

E-mail address: jean-philippe.mandallena@unimes.fr 